\title{
NUCLIDIC MASS RELATIONSHIPS AND MASS EQUATIONS (II)
}

\author{
J. JÄNECKE and B. P. EYNON \\ Department of Physics, Cyclotron Laboratory, The University of Michigan, \\ Ann Arbor, Michigan 48105, USA ${ }^{\dagger}$
}

Received 22 October 1974

\begin{abstract}
Several explicit numerical solutions of generalized nuclidic mass relationships (partial difference equations) have been derived. These solutions are mass equations with about 230 parameters which reproduce more than 1000 experimental masses with $N, Z \geqq 20$ with a standard deviation of about $300 \mathrm{keV}$. The internal consistency of the solutions and other aspects such as the ability to describe the experimental Coulomb displacement energies are explored. The solutions are compared to the transverse and longitudinal mass equations of Garvey and Kelson and the shell-model mass equations of Liran and Zeldes for their reliability to predict masses of very neutron-rich and proton-rich nuclei.
\end{abstract}

\section{Introduction}

Generalized nuclidic mass relationships have been introduced and discussed earlier ${ }^{1,2}$ ). They represent partial difference equations for the nuclear masses with respect to $N$ and $Z$ (or $A$ and $T_{z}$ ). The transverse and longitudinal mass relationships, GK-T and GK-L, of Garvey and Kelson ${ }^{3}$ ) are contained in the general relationships as limiting cases. A necessary condition for an application of the generalized relationships is certain knowledge about the effective neutron-proton interaction $I_{\mathrm{np}}\left(A, T_{z}\right)$ which is defined below. General solutions $M^{*}\left(A, T_{z}\right)$ of the above partial difference equations have been obtained earlier ${ }^{2}$ ). It is the purpose of the present work to present and to discuss explicit numerical solutions $M^{*}\left(A, T_{z}\right)$ based on certain assumptions and theories about $I_{\mathrm{np}}$. The solutions $M^{*}\left(A, T_{z}\right)$ represent manyparameter mass equations which will be distinguished from other (many- or fewparameter) mass equations $M\left(A, T_{z}\right)$ by an asterisk.

The discussion of the new mass equations $M^{*}\left(A, T_{z}\right)$ concentrates on their internal consistency with experimental data which particularly involve the symmetry and Coulomb energy. Emphasis will be given to the question of the reliability of extrapolations into the regions of neutron-rich and proton-rich nuclei. The results will be compared to the predictions from the Garvey-Kelson mass relationships ${ }^{3}$ ), GK-T and GK-L, and to those from the new shell-model mass equations of Liran and Zeldes ${ }^{4}$ ).

All known experimental masses with $N \geqq 20$ and $Z \geqq 20$ were included in the present treatment. The resulting mass equations $M^{*}\left(A, T_{z}\right)$ of sect. 4, however, should

$\uparrow$ Work supported in part by the USAEC. 
only be used for $A \geqq 70$. The dependence of the effective neutron-proton interaction $I_{\mathrm{np}}\left(A, T_{z}\right)$ on shell-model configurations becomes too important in light nuclei and will have to become the subject of further studies. The new mass equations are believed to be more reliable for nuclei far away from the line of beta-stability than known mass equations.

\section{Solutions of generalized nuclidic mass relationships}

The modified transverse and longitudinal nuclidic mass relationships

$$
\begin{aligned}
& M^{*}\left(A, T_{z}+2\right)-M^{*}\left(A, T_{z}\right)+M^{*}\left(A-1, T_{z}+\frac{1}{2}\right)-M^{*}\left(A-1, T_{z}+\frac{3}{2}\right)+M^{*}\left(A+1, T_{z}+\frac{1}{2}\right) \\
& \quad-M^{*}\left(A+1, T_{z}+\frac{3}{2}\right)=I_{\mathrm{np}}\left(A+1, T_{z}+\frac{3}{2}\right)-I_{\mathrm{np}}\left(A+1, T_{z}+\frac{1}{2}\right), \\
& M^{*}\left(A+4, T_{z}\right)-M^{*}\left(A, T_{z}\right)+M^{*}\left(A+1, T_{z}+\frac{1}{2}\right)-M^{*}\left(A+3, T_{z}+\frac{1}{2}\right) \\
& \quad+M^{*}\left(A+1, T_{z}-\frac{1}{2}\right)-M^{*}\left(A+3, T_{z}-\frac{1}{2}\right)=-I_{\mathrm{np}}\left(A+4, T_{z}\right)+I_{\mathrm{np}}\left(A+2, T_{z}\right), \quad \text { (2) }
\end{aligned}
$$

have been introduced and discussed earlier $\left.{ }^{1,2}\right)$. Here, the quantity $I_{\mathrm{pp}}\left(A, T_{z}\right)$ is defined by

$$
\begin{gathered}
I_{\mathrm{np}}\left(A, T_{z}\right)=-M\left(A, T_{z}\right)+M\left(A-1, T_{z}-\frac{1}{2}\right)+M\left(A-1, T_{z}+\frac{1}{2}\right)-M\left(A-2, T_{z}\right) \\
\approx\left(\frac{1}{4} \frac{\partial^{2}}{\partial T_{z}^{2}}-\frac{\partial^{2}}{\partial A^{2}}\right) M\left(A-1, T_{z}\right) .
\end{gathered}
$$

It can be extracted from the experimental masses. However, in order to solve the partial difference eqs. (1) or (2) it is necessary to introduce assumptions or theories about $I_{\mathrm{np}}\left(A, T_{z}\right)$. This is also the case for the generalized nuclidic mass relationships [refs. ${ }^{1,2}$ )] which constitute linear combinations of the difference eqs. (1) and (2). General solutions of the generalized relationships have been derived before ${ }^{2}$ ). They can also be interpreted as those solutions $M^{*}\left(A, T_{z}\right)$ which satisfy eqs. (1) and (2) simultaneously. They consist of a special solution of the inhomogeneous equation and the most general solution of the homogeneous equation. The latter is closely related to the solutions of the Garvey-Kelson nuclidic mass relationships ${ }^{3}$ ).

If it is assumed that $I_{\mathrm{np}}\left(A, T_{z}\right)$ is a function of $A$ only, the generalized nuclidic mass relationships reduce to the GK-T relationship. The most general solution is

$$
M^{*}\left(A, T_{z}\right)=g_{1}(N)+g_{2}(Z)+g_{3}(N+Z) .
$$

Here, $g_{1}(N), g_{2}(Z)$ and $g_{3}(N+Z)$ are arbitrary functions of $N=\frac{1}{2} A+T_{z}$, $Z=\frac{1}{2} A-T_{z}$ and $N+Z=A$, respectively.

If it is assumed that $I_{\mathrm{np}}\left(A, T_{z}\right)$ is a function of $T_{z}$ only, the generalized nuclidic mass relationships reduce to the GK-L relationship. The most general solution is

$$
M^{*}\left(A, T_{z}\right)=f_{1}(N)+f_{2}(Z)+f_{3}(N-Z) .
$$


Here, $f_{1}(N), f_{2}(Z)$ and $f_{3}(N-Z)$ are arbitrary functions of $N=\frac{1}{2} A+T_{z}, Z=\frac{1}{2} A-T_{z}$ and $N-Z=2 T_{z}$, respectively.

If it is assumed that $I_{\mathrm{np}}\left(A, T_{z}\right)$ is constant (separately for even- $A$ and odd- $A$ nuclei), the most general solution of the generalized nuclidic mass relationship is

$$
M^{*}\left(A, T_{z}\right)=\eta_{1}(N-Z)^{2}+\eta_{2} \delta_{\mathrm{oo}}+\eta_{3} \delta_{\mathrm{ee}}+\eta_{4} \delta_{\mathrm{co}}+\eta_{5} \delta_{\mathrm{oc}}+F_{1}(N)+F_{2}(Z) .
$$

Here, $F_{1}(N)$ and $F_{2}(Z)$ are arbitrary functions of $N=\frac{1}{2} A+T_{z}$ and $Z=\frac{1}{2} A-T_{z}$, respectively, and the $\eta_{i}$ are arbitrary constants. The quantity $\delta_{00}$ is one for doubly odd nuclei and is zero otherwise. The quantities $\delta_{\mathrm{ee}}, \delta_{\mathrm{co}}$ and $\delta_{\mathrm{oe}}$ are defined accordingly.

More detailed theories about the $A$ and $T_{z}$ dependence of $I_{\mathrm{np}}$ are implicit in any liquid-drop model, shell model or other mass equation $M\left(A, T_{z}\right)$ and the underlying physical assumptions are those used in the derivation of the mass equation. The quantity $I_{\text {np }}\left(A, T_{z}\right)$ can be extracted $\left.{ }^{1,2,6}\right)$ from any $M\left(A, T_{z}\right)$ by the use of eq. (3). It should be noted that except for small Coulomb energy contributions the quantity $I_{\mathrm{np}}\left(A, T_{z}\right)$ must satisfy $I_{\mathrm{np}}\left(A,-T_{z}\right) \approx I_{\mathrm{np}}\left(A,+T_{z}\right)$ because of charge-symmetry of nuclear forces. It is for this reason that eq. (5), for example, is not valid on both sides of the $N=Z$ line for given functions $f_{i}(k)$.

Given $I_{\mathrm{np}}\left(A, T_{z}\right)$ from a mass equation $M\left(A, T_{z}\right)$, a general solution $\left.{ }^{2}\right) M^{*}\left(A, T_{z}\right)$ of the generalized nuclidic mass relationships is

with

$$
M^{*}\left(A, T_{z}\right)=M\left(A, T_{z}\right)+\Delta M\left(A, T_{z}\right),
$$

$$
\Delta M\left(A, T_{z}\right)=\eta_{1}(N-Z)^{2}+\eta_{2} \delta_{\mathrm{oo}}+\eta_{3} \delta_{\mathrm{ee}}+\eta_{4} \delta_{\mathrm{eo}}+\eta_{5} \delta_{\mathrm{oe}}+F_{1}(N)+F_{2}(Z)
$$

Here, $M\left(A, T_{z}\right)$ represents a special solution of the inhomogeneous eqs. (1) and (2) and $\Delta M\left(A, T_{z}\right)$ represents the most general solution of the homogeneous equations. The terms $F_{1}(N)$ and $F_{2}(Z)$ are again arbitrary functions of $N=\frac{1}{2} A+T_{z}$ and $Z=\frac{1}{2} A-T_{z}$, respectively, and the $\eta_{i}$ are arbitrary constants. It should be emphasized that in eq. (7) use is made only of $I_{\mathrm{np}}\left(A, T_{z}\right)$ implicitly contained in $M\left(A, T_{z}\right)$ and any other mass equation with the same $I_{\text {np }}\left(A, T_{z}\right)$ will result in an identical solution $M^{*}\left(A, T_{z}\right)$.

The constants $\eta_{i}$ and functions $F_{1}(N)$ and $F_{2}(Z)$ (as well as the earlier functions $g_{i}(k)$ and $\left.f_{2}(k)\right)$ can be constructed for any $M\left(A, T_{z}\right)$ from a $\chi^{2}$ minimization to the experimental masses. The solution of the ensuing system of 200-250 linear equations for the 200-250 parameters is a correction term $\Delta M\left(A, T_{z}\right)$ which will convert any few-parameter mass equation $M\left(A, T_{z}\right)$ into a many-parameter mass equation $M^{*}\left(A, T_{z}\right)$ with a generally greatly increased accuracy for reproducing the experimental masses.

Another aspect of the above treatment is that it provides insight into the structure and possible shortcomings of a mass equation $M\left(A, T_{z}\right)$ (referred to as test 3 in ref. $\left.{ }^{2}\right)$ ). Clearly, the "better" $M\left(A, T_{z}\right)$ is, the smaller $\Delta M\left(A, T_{z}\right)$ will be. Additional information is obtained if the various contributions to $\Delta M$ are considered separately. A finite coefficient $\eta_{1}$ points to a deficiency in the description of the nuclear symmetry 
energy. In the Bethe-Weizsäcker liquid-drop model equation, for example, one obtains a corrected energy

$$
E_{\mathrm{sym}}^{*}=\left(a_{\mathrm{sym}}+\eta_{1} A\right)(N-Z)^{2} / A .
$$

A finite value of $\eta_{1}$ shows the inadequacy of a constant symmetry energy coefficient $a_{\text {sym }}$. Finite values of $\eta_{2}$ and $\eta_{3}$ in particular but also of $\eta_{4}$ and $\eta_{5}$ point to the need for a neutron-proton pairing energy term. Furthermore, the functions $F_{1}(N)$ and $F_{2}(Z)$ give additional information about nuclear shell effects and the Coulomb energy.

A possible decomposition of $\Delta M$ is

$$
\Delta M\left(A, T_{z}\right)=\Delta M_{\text {nucl }}(N)+\Delta M_{\text {nucl }}(Z)+\Delta M_{\text {Coul }}(Z)+\Delta M_{\text {sym }}(N, Z)+\Delta M_{\text {pair }}(N, Z),
$$

with

$$
\begin{gathered}
\Delta M_{\text {nucl }}(k)=F_{1}(k), \\
\Delta M_{\text {Coul }}(Z)=F_{2}(Z)-F_{1}(Z), \\
\Delta M_{\text {sym }}(N, Z)=\eta_{1}(N-Z)^{2}, \\
\Delta M_{\text {pair }}(N, Z)=\eta_{2} \delta_{\infty}+\eta_{3} \delta_{\mathrm{ce}}+\eta_{4} \delta_{\mathrm{co}}+\eta_{5} \delta_{\mathrm{oc}} .
\end{gathered}
$$

The correction $\Delta M$ is uniquely determined from the $\chi^{2}$ minimization. This is not true for the individual contributions (except for $\Delta M_{\text {sym }}$ ) since the replacements

$$
\begin{aligned}
F_{1}(N) & \rightarrow F_{1}(N)+c_{1}+(-1)^{N} c_{2}, \\
F_{2}(Z) & \rightarrow F_{2}(Z)-c_{1}+(-1)^{z} c_{3}, \\
& \eta_{2} \rightarrow \eta_{2}+c_{2}+c_{3}, \\
& \eta_{3} \rightarrow \eta_{3}-c_{2}-c_{3}, \\
& \eta_{4} \rightarrow \eta_{4}-c_{2}+c_{3}, \\
\eta_{5} & \rightarrow \eta_{5}+c_{2}-c_{3},
\end{aligned}
$$

will lead to identical results for arbitrary constants $c_{1}, c_{2}$ and $c_{3}$. Without loss of generality one can therefore assume $F_{1}\left(N_{\text {ref }}\right)=F_{2}\left(Z_{\text {ref }}\right), \eta_{2}-\eta_{3}=0$ and $\eta_{4}+\eta_{5}=0$. Here, $N_{\text {ref }}$ and $Z_{\text {ref }}$ are two arbitrarily chosen reference values. The splitting of the mass surface for odd- $A$ nuclei due to the term $\eta_{4}\left(\delta_{\mathrm{eo}}-\delta_{\mathrm{eo}}\right)$ is very small (see below). It is therefore further assumed that $\eta_{4}=0$. The above remarks show that nuclear and Coulomb contributions are determined except for added constants, namely $F_{\text {nucl }}(k)+c$ and $F_{\text {Coul }}-2 c$, respectively.

Strong correlations were observed between the Coulomb energy and symmetry energy correction terms. This led to the conclusion that the Coulomb energy correction term $\Delta E_{\text {Coul }}=F_{2}(Z)-F_{1}(Z)$ represents a useful correction only if the symmetry energy is well described by the term included in the mass equation together with the correction term $\Delta M_{\text {sym }}=\eta_{1}(N-Z)^{2}$. Adding correction terms $\Delta M$ will always lead 
to considerable decreases in the standard deviation $\sigma_{\mathrm{m}}$ for reproducing the experimental masses. The standard deviation $\sigma_{\mathrm{o}}$ for reproducing the experimental Coulomb displacement energies, on the other hand, sometimes increased greatly indicating that improvements in $\sigma_{\mathrm{m}}$ can be achieved by simultaneously misrepresenting the symmetry energy and the Coulomb energy. Extrapolations into the regions of neutron-rich or proton-rich nuclei will, of course, be greatly affected by such misrepresentations.

The $\chi^{2}$ minimizations were therefore carried out using three different procedures. The correction term $\Delta M$ of eq. (7) was constructed (A) with no constraint; (B) with the constraint $\Delta M_{\text {Coul }} \equiv 0$, i.e., $F_{1}(k)=F_{2}(k)$, and (C) with the constraint $\Delta M_{\text {sym }} \equiv 0$, i.e., $\eta_{1}=0$. In case $B$ the number of adjusted parameters is greatly reduced.

Results were obtained for 16 different assumptions and theories for $I_{\mathrm{np}}$. They are listed in table 1 and will be described briefly in the following.

TABLE 1

List of mass equations

\begin{tabular}{|c|c|}
\hline GK-T & Garvey-Kclson transverse ${ }^{3}$ ). \\
\hline GK-L & Garvey-Kelson longitudinal ${ }^{3}$ ). \\
\hline CON & $I_{\mathrm{np}}=$ const. \\
\hline $\mathbf{Z}$ & Zeldes et al. ${ }^{\mathbf{5}}$ ) shell model. \\
\hline LZ1 & Liran and Zeldes ${ }^{4}$ ) shell model, seniority scheme. \\
\hline $\mathbf{L Z 2}$ & Liran and Zeldes ${ }^{4}$ ) shell model, seniority scheme with deformation corrections. \\
\hline BW1 & $\begin{array}{l}\text { Bethe-Weizsäcker liquid-drop model, parameters optimized for minimum } \sigma_{m} \\
\left(b_{c}=-a_{c}\right)\end{array}$ \\
\hline BW2 & $\begin{array}{l}\text { Rethe-Weizsücker liquid-drop model, parameters } a_{c}, b_{\mathrm{c}} \text { optimized for minimum } \sigma_{c} \text {, } \\
\text { remaining parameters optimized for minimum } \sigma_{\mathrm{m}} \text {. }\end{array}$ \\
\hline BW3-5 & $\begin{array}{l}\text { Bethe-Weizsâcker liquid-drop model, parameters } a_{\mathrm{C}}, b_{\mathrm{c}} \text { optimized for minimum } \sigma_{\mathrm{C}} \text {, } \\
\text { remaining parameters including } \eta_{1}, \eta_{2}, F_{1}(N) \text { and } F_{2}(Z) \text { optimized for minimum } \\
\sigma_{\mathrm{m}} \text {; cases A, B and C, respectively. }\end{array}$ \\
\hline MS & Myers and Swiatecki droplet model [ref. ${ }^{8}$ ), parameters of ref. ${ }^{9}$ )]. \\
\hline MSL & Myers and Swiatecki droplet model [ref. ${ }^{8}$ ), parameters of ref. ${ }^{10}$ )]. \\
\hline $\mathbf{C}$ & Cameron et al. ") liquid-drop model. \\
\hline Cs & Cameron et al. ${ }^{11}$ ) liquid-drop model with shell and pairing corrections. \\
\hline $\mathbf{S}$ & Seeger ${ }^{12}$ ) liquid-drop model. \\
\hline
\end{tabular}

The abbreviated notations GK-T, GK-L and CON refer to the assumptions $I_{\mathrm{np}}=I_{\mathrm{np}}\left(T_{z}\right), I_{\mathrm{np}}=I_{\mathrm{np}}(A)$ and $I_{\mathrm{np}}=$ constant, respectively. The parameters of ref. ${ }^{3}$ ) were used. The notations $Z, L Z 1$ and $L Z 2$ refer to the shell-model theories for $I_{\mathrm{np}}$ implicit in the mass equations of Zeldes, Grill and Simievic ${ }^{5}$ ) and of Liran and Zeldes ${ }^{4}$ ). The label $Z$ refers to the earlier version ${ }^{5}$ ) while $L Z 1$ and $L Z 2$ refer to the recent work ${ }^{4}$ ). The first one, $L Z 1$, is essentially based on the seniority coupling scheme. The second one, LZ2, includes additional empirical correction terms to account for deformation effects and core excitations.

The remaining cases refer to the theories for $I_{\mathrm{np}}$ implicit in liquid-drop model mass equations. The notations BW1 through BW5 stand for the Bethe-Weizsäcker equation with the five parameter sets of table 2 . The equation is written in a slightly 
TABLE 2

Parameters for the Bethe-Weizsäcker equation (in keV)

\begin{tabular}{|c|c|c|c|c|c|}
\hline & BW1 & BW2 & BW3 & BW4 & BW5 \\
\hline$a_{v}$ & 16313 & 15326 & $\left.15326^{a}\right)$ & $\left.15326^{\mathrm{a}}\right)$ & $\left.15326^{a}\right)$ \\
\hline$a_{8}$ & 19436 & 17257 & $\left.17257^{a}\right)$ & $\left.17257^{a}\right)$ & $17257^{\text {a) }}$ \\
\hline$a_{\text {sym }}$ & 23537 & 21161 & 13878 & 18156 & 18993 \\
\hline$a_{\mathrm{C}}$ & 763 & $\left.695^{b}\right)$ & $\left.695^{b}\right)$ & $\left.695^{b}\right)$ & $\left.695^{b}\right)$ \\
\hline$b_{\mathrm{c}}$ & -763 & $\left.-2736^{b}\right)$ & $\left.-2736^{b}\right)$ & $\left.-2736^{b}\right)$ & $\left.-2736^{b}\right)$ \\
\hline$a_{p}^{(1)}$ & 36949 & 32077 & $\left.32077^{a}\right)$ & $\left.32077^{\circ}\right)$ & $\left.32077^{a}\right)$ \\
\hline$a_{\mathrm{p}}^{(2)}$ & 5044 & $\left.5044^{a}\right)$ & $\left.5044^{a}\right)$ & $\left.5044^{3}\right)$ & $\left.5044^{*}\right)$ \\
\hline$a_{p}^{(3)}$ & $\left.0^{a}\right)$ & $0^{\text {a) }}$ & $\left.0^{a}\right)$ & $\left.0^{a}\right)$ & $\left.0^{a}\right)$ \\
\hline$\eta_{1}$ & & & 52.4 & 17.8 & 0.0 \\
\hline$\eta_{2}$ & & & 24.9 & 25.0 & 25.6 \\
\hline
\end{tabular}

a) Not adjusted.

b) From ref. ${ }^{7}$ ).

modified form as

$$
\begin{aligned}
M(N, Z)= & M_{\mathrm{n}} N+M_{\mathrm{H}} Z-a_{\mathrm{v}} A+a_{\mathrm{s}} A^{\xi}+a_{\mathrm{sym}} \frac{(N-Z)^{2}+2|(N-Z)|}{A} \\
& +\frac{a_{\mathrm{c}} Z^{2}+b_{\mathrm{c}} Z}{A^{\frac{Z}{3}}}-\frac{\delta_{\mathrm{ee}}\left(a_{\mathrm{p}}^{(1)}+a_{\mathrm{p}}^{(2)}\right)+\left(\delta_{\mathrm{eo}}-\delta_{\mathrm{oe}}\right) a_{\mathrm{p}}^{(3)}+\delta_{\mathrm{oo}}\left(-a_{\mathrm{p}}^{(1)}+a_{\mathrm{p}}^{(2)}\right)}{A^{Z}} .
\end{aligned}
$$

The symmetry energy term is modified to conform to the $T(T+1)$ dependence obtained in shell-model equations. The pairing energy term is generalized by introducing the term with $a_{\mathrm{p}}^{(2)}$ to allow for a different separation between the doubly even/odd- $A$ and doubly odd/odd- $A$ mass surfaces. Furthermore, the term with $a_{\mathrm{p}}^{(3)}$ removes the degeneracy between the even-odd and odd-even mass surfaces for odd- $A$ nuclei. Only the term with $a_{\mathrm{p}}^{(1)}$ is generally included. Since $M(N, Z)$ of eq. (12) depends linearly on the parameters, a $\chi^{2}$ minimization leads to a simple system of equations for the parameters which has a unique solution. The solution for BW1 is based on the assumption $b_{\mathrm{C}}=-a_{\mathrm{C}}$ which is often made. Also, the pairing term with $a_{\mathfrak{p}}^{(3)}$ was set to zero because there was practically no difference between this solution and the solution where $a_{\mathrm{p}}^{(3)}$ was permitted to differ from zero $\left(a_{\mathrm{p}}^{(3)} / a_{\mathrm{p}}^{(1)} \approx 0.05\right)$. A different exponent of 0.5 in the $A$-dependence of the pairing terms had practically no effect on the other coefficients and on the standard deviation. The parameter set for BW2 was obtained in practically the same way except that $a_{\mathrm{C}}$ and $b_{\mathrm{c}}$ were fixed at those values which independently minimize the experimental Coulomb displacement energies ${ }^{7}$ ) with a standard deviation of $\sigma_{\mathrm{C}}=84 \mathrm{keV}$. The subsequent determination of the coefficients $\eta_{1}$ and $\eta_{2}$ and the functions $F_{1}(N)$ and $F_{2}(Z)$ for the solutions $M^{*}\left(A, T_{z}\right)$ (conditions $\mathrm{A}, \mathrm{B}$ or $\mathrm{C}$ ) was done separately. The pairing term $a^{(1)}$ was set to zero because the functions $F_{1}(N)$ and $F_{2}(Z)$ reproduce the $\mathrm{n}-\mathrm{n}$ and $\mathrm{p}-\mathrm{p}$ pairing energies more correctly than the term $\pm a_{\mathrm{p}}^{(1)} A^{-7}$. The three remaining solutions $\mathrm{BW} 3, \mathrm{BW} 4$ and $\mathrm{BW} 5$ were obtained under conditions 
TABLB 3

Standard deviations $\sigma_{\mathrm{m}}$ for the experimental masses and $\sigma_{\mathrm{C}}$ for the Coulomb displacement energies mass relationships obtained

\begin{tabular}{|c|c|c|c|c|c|c|}
\hline & GK-T & GK-L & $\mathrm{CON}$ & $\mathrm{Z}$ & $\mathbf{L Z 1}$ & $\mathrm{LZ2}$ \\
\hline Number of exp. masses $n$ & 1040 & 1041 & 1059 & 1058 & 1053 & 1053 \\
\hline & \multicolumn{6}{|c|}{ Mass equation $M\left(A, T_{z}\right)$} \\
\hline Number of parameters $p$ & 484 & 275 & & $\left.320^{x}\right)$ & $\left.90^{\circ}\right)$ & $\left.340^{\prime \prime}\right)$ \\
\hline Standard deviation $\sigma_{\mathrm{m}}$ & 171 & 268 & & 188 & 761 & 167 \\
\hline Standard deviation $\sigma_{\mathfrak{C}}$ & 2598 & b) & & b) & $\left.129^{\circ}\right)$ & $\left.65^{\circ}\right)$ \\
\hline
\end{tabular}

(A) Solution $M^{*}\left(A, T_{z}\right)$; no constraint

Number of parameters $p$

Standard deviation $\sigma_{m}$ Standard deviation $\sigma_{\mathrm{C}}$

Coefficient $\eta_{2}$

Coefficient $\eta_{2}$

Quantity $F_{\text {Coul }}(100)$

Number of parameters $p$

Standard deviation $\sigma_{\mathrm{m}}$

Standard deviation $\sigma_{\mathrm{C}}$

Coefficient $\eta_{1}$

Coefficient $\eta_{2}$

Quantity $F_{\text {Coul }}(100)$

Number of parameters $p$

Standard deviation $\sigma_{\mathrm{m}}$

Siandard deviation $\sigma_{\mathrm{C}}$

Coefficient $\eta_{1}$

Coefficient $\eta_{2}$

Quantity $F_{\text {Coul }}(100)$

$\begin{array}{cc}220 & 310 \\ 662 & 244 \\ 5479 & \left.368^{\circ}\right) \\ 181.1 & -2.8 \\ -113.0 & -29.5 \\ 1335 & -19\end{array}$

(B) Solution $M^{*}\left(A, T_{z}\right)$; constraint $F_{1}(k)=F_{2}(k)$

230
333
$\left.129^{\circ}\right)$
0.2
-37.1
0

(C) Solution $M^{*}\left(A, T_{z}\right)$; constraint $\eta_{\mathrm{t}}=0$

The experimental masses with $N \geqq 20$ and $Z \geqq 20$ from the 1971 atomic mass evaluation ${ }^{13}$ ) were used

a) The numbers of parameters actually different from zero are 201,87 and 144 , respectively.

c) Region $Z>82$ and discontinuities at all shell closurcs are excluded.

e) Calculated from the experimental displacement energies for spherical nuclei with $Z \geqq 20$ and the

$\mathrm{A}, \mathrm{B}$ or $\mathrm{C}$ by simultaneously optimizing $a_{\text {sym }}$ and the coefficients $\eta_{1}$ and $\eta_{2}$ and the functions $F_{1}(N)$ and $F_{2}(Z)$. Thus, BW3 and BW4 allow for a linear increase with $A$ of the symmetry energy coefficient.

Results for the droplet-model mass equation of Myers and Swiatecki ${ }^{8}$ ) are listed as MS and MSL. The first one uses the parameter set of Myers and Swiatecki ${ }^{9}$ ). The second one is for the parameter set of Ludwig et al. ${ }^{10}$ ). Results for the liquid-drop model mass equation of Cameron ${ }^{11}$ ) are given as $\mathrm{C}$ and $\mathrm{Cs}$. The second equation includes the empirical shell and pairing correction terms introduced by the authors ${ }^{11}$ ) on the assumption $C(N, Z)=C_{1}(N)+C_{2}(Z)$. The structure of these corrections is almost identical to the ones derived in the present work under condition $C$. The 
for the mass equations $M\left(A, T_{z}\right)$ of table 1 and for the solutions $M^{*}\left(A, T_{z}\right)$ of the generalized nuclidic under three conditions $\mathrm{A}, \mathrm{B}$, and $\mathrm{C}$

\begin{tabular}{|c|c|c|c|c|c|c|c|c|c|}
\hline BW1 & BW2 & BW3 & BW4 & BW5 & MS & MSL & $\mathrm{C}$ & Cs & $\mathbf{S}$ \\
\hline 1059 & 1059 & 1059 & 1059 & 1059 & 1059 & 1059 & 1059 & 1057 & 1059 \\
\hline 6 & 7 & & & & 12 & 12 & 17 & 233 & 12 \\
\hline 2801 & 3576 & & & & 3171 & 6877 & 2767 & 316 & 3995 \\
\hline 1977 & $\left.84^{d}\right)$ & & & & $\left.171^{\circ}\right)$ & $\left.192^{\circ}\right)$ & 970 & 960 & 271 \\
\hline 225 & 226 & 226 & & & 231 & 231 & 236 & & 231 \\
\hline 472 & 385 & 238 & & & 259 & 291 & 261 & & 260 \\
\hline 1560 & 884 & 1082 & & & $\left(160^{\circ}\right)$ & $\left.1184^{\circ}\right)$ & 1747 & & 664 \\
\hline-27.3 & -6.2 & 52.4 & & & 19.2 & 5.4 & 8.4 & & 4.1 \\
\hline 22.9 & 23.5 & 24.9 & & & -122.9 & -124.4 & -100.0 & & -110.4 \\
\hline-175 & -4 & +15 & & & 129 & 103 & 49 & & 36 \\
\hline 145 & 146 & & 146 & & 151 & 151 & 156 & & 151 \\
\hline 920 & 1036 & & 536 & & 519 & 793 & 510 & & 483 \\
\hline 1977 & $\left.84^{d}\right)$ & & $\left.84^{d}\right)$ & & $\left.171^{\mathrm{c}}\right)$ & $\left.192^{\mathrm{e}}\right)$ & 970 & & 271 \\
\hline 2.4 & -0.9 & & 17.8 & & 0.3 & -6.9 & 0.4 & & -1.2 \\
\hline 56.1 & 63.3 & & 25.0 & & $\ldots-113.9$ & -91.6 & -94.3 & & -99.3 \\
\hline 0 & 0 & & $\mathbf{0}$ & & 0 & 0 & 0 & & 0 \\
\hline 224 & 225 & & & 225 & 230 & 230 & 235 & & 230 \\
\hline 543 & 389 & & & 338 & 320 & 322 & 274 & & 263 \\
\hline 2057 & 778 & & & 1267 & $\left.399^{\circ}\right)$ & $\left.971^{\circ}\right)$ & 977 & & 420 \\
\hline 0 & 0 & & & 0 & 0 & 0 & 0 & & 0 \\
\hline 18.9 & 22.6 & & & 25.6 & -120.0 & -120.0 & -98.8 & & -109.8 \\
\hline 3.7 & 36.6 & & & -72.0 & 3.2 & 12.0 & -5.5 & & 9.6 \\
\hline
\end{tabular}

in the $\chi^{2}$ minimization. Values of $\sigma, \eta$ in $\mathrm{keV}, F_{\text {Cou } 1}$ in $\mathrm{MeV}$.

b) Cannot be calculated.

d) Standard deviation for $\Delta E$ (exp) $-\Delta E_{\text {cout }}$ (calc).

(combined electrostatic and nucicar) displacement energies from the equation.

line labelled $S$, finally, is based on the liquid-drop model mass equation of Seeger ${ }^{12}$ ). The basic results obtained from the $\chi^{2}$ minimizations for the slightly more than 1000 experimental masses are contained in table 3 . The experimental masses from the 1971 atomic mass evaluation ${ }^{13}$ ) were used but only masses with $N \geqq 20$ and $Z \geqq 20$ were included. Lighter nuclei will have to be treated separately because the liquid-drop equations are not adequate. Table 3 lists for all equations of table 1 as well as for the solutions obtained under conditions $A, B$ and $C$ the number of experimental masses $n$ and the number of parameters $p$. Solutions for GK-T, GK-L, $Z$ and LZ2 were obtained but are excluded from the table because of the large number of parameters. Also given are the standard deviations $\sigma_{m}$ for the experimental masses 
and $\sigma_{\mathrm{C}}$ for the Coulomb displacement energies along the line of $\beta$-stability. Unless noted otherwise in the table, it was considered equivalent (and simpler) to use for the comparison the empirical equation

$$
\Delta E_{\mathrm{c}}=\frac{2 a_{\mathrm{c}}\left(Z_{-}+0.5\right)+b_{\mathrm{c}}}{A^{\frac{1}{3}}}
$$

instead of the experimental displacement energies. Eq. (13) with the parameters of ref. ${ }^{7}$ ) reproduces the experimental values with a standard deviation of $\sigma_{\mathrm{C}}=84 \mathrm{keV}$. Additional lines in table 3 give the values of $\eta_{1}, \eta_{2}$ and $F_{\text {Coul }}(100)$.

\section{Discussion}

The number of parameters used in the various equations and procedures varies from six or seven for the Bethe-Weizsäcker equation to 484 for the transverse Garvey-Kelson equation. This variation has to be considered when comparing the standard deviations $\sigma_{m}$ which range from a few $\mathrm{MeV}$ for the former to less than $200 \mathrm{keV}$ for the latter. A possible classification is $p<25$ for few-parameter equations and $p \geqq 25$ for many-parameter equations. All liquid-drop model equations are few-parameter equations. The majority of many-parameter equations have 220 to 240 parameters (solutions $A$ and $C$ ), a few have about 150 (solutions $B$ ) and about 300 parameters, respectively, and one each has $<100$ and $>450$ parameters.

Fig. 1 shows a plot of the quantity $\sigma_{m}^{2} /(n-p)$ as a function of $p$. The square of the standard deviation $\sigma_{\mathrm{m}}$ divided by the number of degrees of freedom $f=n-p$ is proportional to $\chi^{2} /(n-p)$ and thus characterizes the goodness of fit over the domain of measured masses. The goodness of fit improves with increasing numbers of parameters $p$ approximately inversely to $p$. The thin line represents $\sigma_{\mathrm{m}}^{2} /(n-p) \propto p^{-1.3}$. Little, however, can be inferred from this quantity about the expected accuracy of predictions for masses of nuclei outside the domain of measured masses except for extrapolations which reach only a few steps outside.

Other aspects have to be taken into consideration. For example, most equations can be used to calculate Coulomb displacement energies. The standard deviations $\sigma_{\mathrm{C}}$ from the comparison with the experimental values are included in table 3 . The smallest deviation $\sigma_{\mathrm{m}}$ exists for the equation GK-T. On the other hand the calculated Coulomb displacement energies exhibit systematic deviations from the experimental values [see fig. 7 of ref. ${ }^{3}$ )] which leads to one of the biggest $\sigma_{\mathrm{C}}$ of all few- and manyparameter equations. Furthermore, the Coulomb energies are functions of $Z$ only. The isotope shift coefficient $\gamma_{N}$ for the nuclear charge radius is therefore identically equal to zero in disagreement with the data. Because of the strong correlation (see below) between Coulomb and symmetry energies, it is concluded that predictions for nuclei far away from the line of $\beta$-stability have to be considered with caution. Similar conclusions have been reached earlier by Sorensen ${ }^{14}$ ). 


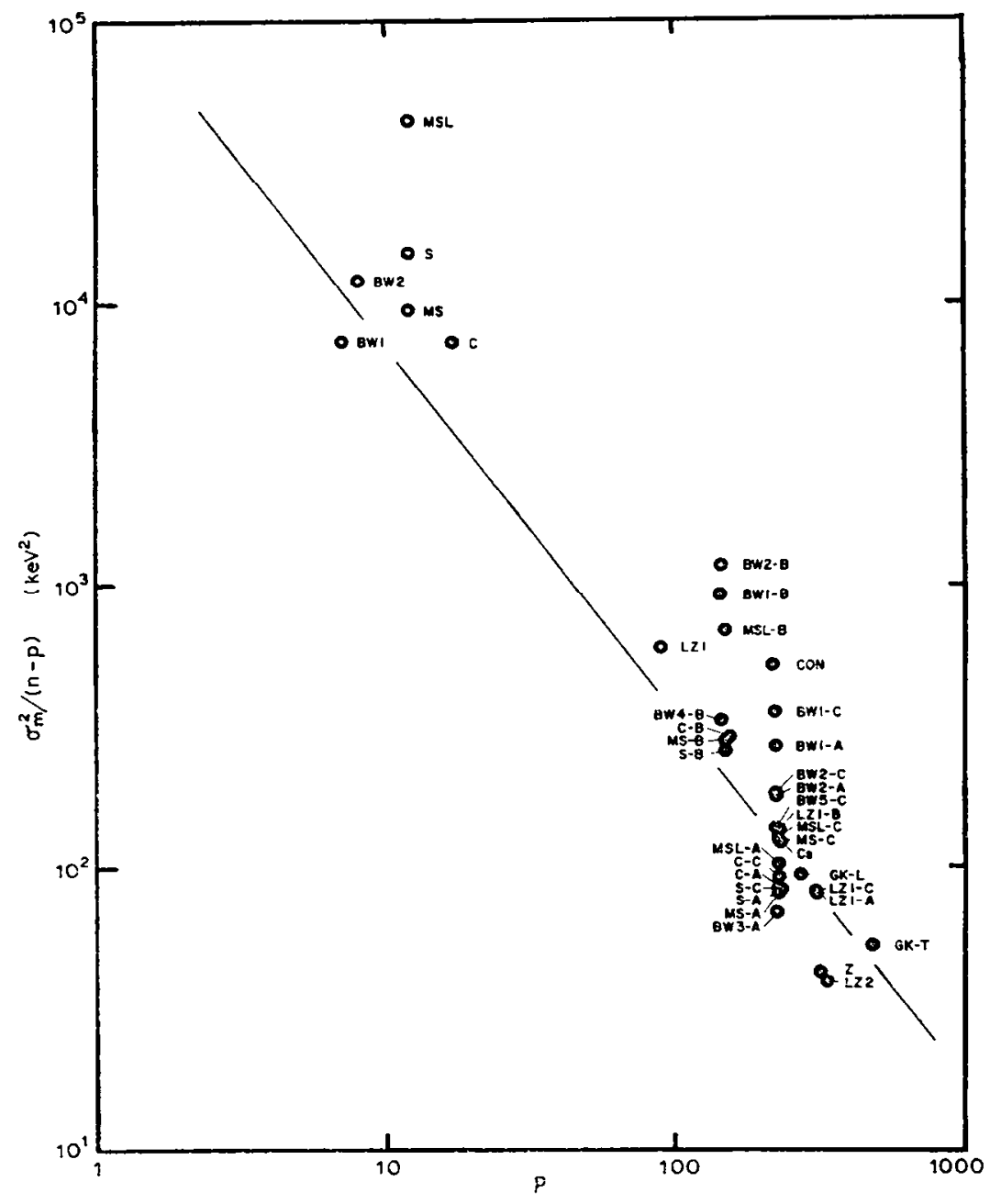

Fig. 1. Plot of the quantity $\sigma_{\mathrm{m}}{ }^{2} /(n-p)$ as a function of the number of parameters $p$ for the 37 mass equations and solutions of generalized nuclidic mass relationships of table 3 . The thin line represents $\sigma_{\mathrm{m}}{ }^{2} /(n-p) \propto p^{-1.3}\left(\sigma_{\mathrm{m}}=\right.$ standard deviation; $n-p=$ number of degrees of freedom).

It appears that only Liran and Zeldes ${ }^{4}$ ) in the equations LZ1 and LZ2 have explicitly included Coulomb energy data to independently determine Coulomb energy coefficients. The influence of the strong Coulomb energy/symmetry energy correlations on extrapolations can thereby be reduced. The remaining part of the equation has to satisfy charge symmetry of nuclear forces. Their expression

$$
\Delta E_{\mathrm{C}}=\left(\frac{A_{1}}{A}\right)^{\tau}\left(\beta_{\mathrm{C}}(2 p+1)+\gamma_{\mathrm{C}}\right)
$$


is in excellent agreement with the data within all shells (with the possible exception of the region beyond ${ }^{208} \mathrm{~Pb}$ ), but serious discrepancies of up to $20 \mathrm{MeV}$ exist at all magic neutron and proton shell closures.

The simple Coulomb energy expression $E_{\mathrm{C}}=a_{\mathrm{C}} Z(Z-1) A^{-3}$ of the BetheWeizsäcker equation BW1 has been replaced by the two-parameter Coulomb energy expression $E_{\mathrm{C}}=\left(a_{\mathrm{C}} Z^{2}+b_{\mathrm{C}} Z\right) A^{-\frac{1}{3}}$ in BW2-BW5. The energy differences $\Delta E_{\mathrm{C}}=$ $\left(2 a_{C}\left(Z_{<}+0.5\right)+b_{C}\right) A^{-\frac{1}{3}}$ describe $\left.{ }^{7}\right)$ the experimental Coulomb displacement energies with a greatly reduced standard deviation of $\sigma_{\mathrm{C}}=84 \mathrm{keV}$.

The droplet model mass equation MS and the solutions MS-A, MS-B and MS-C of the generalized nuclidic mass relationships which are based on $I_{\mathrm{np}}$ implicitly contained in MS will be used as examples to demonstrate the correlation between Coulomb and symmetry energy. Table 3 lists for MS values of $\sigma_{\mathrm{m}}=3171 \mathrm{keV}$ and $\sigma_{\mathrm{c}}=171 \mathrm{keV}$. [The displacement energies calculated from the droplet model MS contain electrostatic and nuclear contributions ${ }^{15}$ ).] The unrestricted solution MS-A has a vastly improved $\sigma_{\mathrm{m}}=259 \mathrm{keV}$ but at the same time a much poorer $\sigma_{\mathrm{C}}=1160 \mathrm{keV}$. Also, the coefficient $\eta_{1}=19.2 \mathrm{keV}$ of the correction term $\Delta M_{\text {sym }}=\eta_{1}(N-Z)^{2}$ is rather big indicating that the smallness of $\sigma_{\mathrm{m}}$ is accomplished by a delicate misrepresentation of $\Delta M_{\mathrm{Coul}}$ as well as $\Delta M_{\mathrm{sym}}$. Therefore, the unrestricted solutions A cannot necessarily be expected to result in reliable extrapolations away from the line of $\beta$-stability. The restricted solutions $\mathrm{B}$ and $\mathrm{C}$ do not have this shortcoming because of the constraints $\Delta E_{\mathrm{Coul}} \equiv 0$ or $\Delta E_{\mathrm{sym}} \equiv 0$. The original mass equations and the associated solutions $B$ have always the same standard deviation $\sigma_{\mathrm{C}}$. The solutions $\mathrm{C}$, however, were found to have slightly increased standard deviations $\sigma_{\mathrm{C}}$ (except for S-C) suggesting slight mistepresentations of the symmetry energy in the original mass cquations. It should be noted that almost all mass equations (except LZ1, LZ2, BW2 to BW5 and the associated solutions B) display systematic rather than random differences between the predicted and experimental Coulomb displacement energies.

Fig. 2 shows as typical examples the functions $F_{\text {nucl }}(k) \equiv F_{1}(k)$ and $F_{\text {Coul }}(k) \equiv$ $F_{2}(k)-F_{1}(k)$ for the solutions MS-A, MS-B and MS-C. The Coulomb energy correction term $F_{\text {Coul }}(Z)$ for MS-A $\left(F_{\text {Coul }}(20)=0\right.$ is arbitrarily chosen) increases strongly with $Z$ and reaches a value of $129 \mathrm{MeV}$ for $Z=100$ which is responsible for the strongly increased standard deviation $\sigma_{\mathrm{C}}$. For the solution MS-B we have $F_{\text {Coul }}(Z) \equiv 0$ and for MS-C the correction term $F_{\text {Coul }}(Z)$ remains quite small confirming the earlier remarks.

The functions $F_{\text {nucl }}(k)$ display very pronounced shell and pairing effects. The oscillations due to the nuclear $\mathrm{n}-\mathrm{n}$ and $\mathrm{p}$-p pairing energies replace those described by the pairing term $\pm a_{\mathrm{p}} A^{-\frac{3}{4}}$ (or similar) which is always removed from $M\left(A, T_{z}\right)$ before the functions $F_{1}(N)$ and $F_{2}(Z)$ are constructed. The depression of $F_{\text {nucl }}(k)$ in the region $k=100$ is apparently due to deformation effects. A reasonable extrapolation of the functions $F_{\text {nucl }}(k)$ and $F_{\text {Coul }}(k)$ should make it possible to predict masses of superheavy nuclei. 


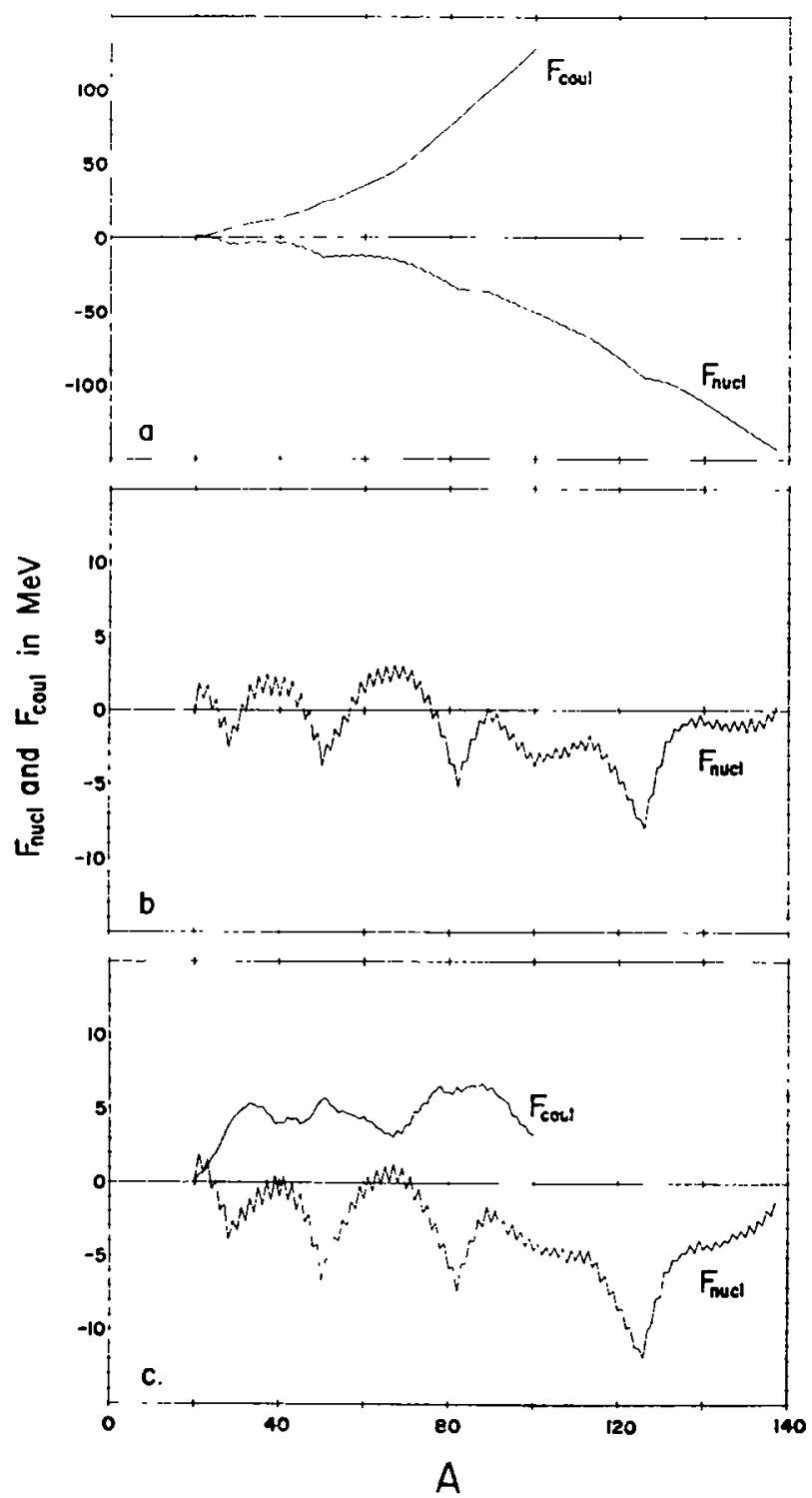

Fig. 2. Plot of the functions $F_{\text {nucl }}(k) \equiv F_{1}(k)$ and $F_{\text {Coul }}(k) \equiv F_{2}(k)-F_{1}(k)$ obtained for the solutions MS-A (no constraint), MS-B (constraint $F_{1}(k)=F_{2}(k)$ ) and MS-C (constraint $\eta_{1}=0$ ) based on $I_{\mathrm{np}}$ from the droplet model mass equation MS of Myers and Swiatecki ${ }^{8,9}$ ).

Values of $\eta_{2} \approx-110 \mathrm{keV}$ (see table 3 ) indicate that no n-p pairing energy term of the type $a_{\mathrm{p}}^{(2)} A^{-\frac{3}{4}} \delta_{\text {even } A}$ has been included in the original mass equation $M\left(A, T_{z}\right)$. The effective neutron-proton interaction $I_{\text {np }}$ derived from such a mass equation will therefore not reproduce the even- $A /$ odd $-A$ splitting ${ }^{2}$ ) of $I_{\text {np }}$. 
The aforementioned discussion suggests that in constructing a mass equation $M\left(A, T_{z}\right)$ it is advisable to determine the Coulomb energy parameters independently from Coulomb energy data. If both Coulomb and symmetry energies are represented well by $M\left(A, T_{z}\right)$, the solutions $M^{*}\left(A, T_{z}\right)$ obtained under conditions $\mathrm{A}, \mathrm{B}$ and $\mathrm{C}$ should give similar standard deviations $\sigma_{\mathrm{m}}$ and $\sigma_{\mathrm{C}}$ and condition B with the smallest number of parameters should be given the preference. An inspection of table 3 shows that such a situation does not exist for any of the listed mass equations. The solutions obtained under condition $\mathrm{C}$ are therefore considered at present to give the most reliable results even though the solutions obtained under condition $A$ which contain only a single additional parameter have smaller standard deviations $\sigma_{\mathrm{m}}$.

To substantiate and illustrate some of the above arguments and comments about extrapolation, it was considered worthwhile to simply plot the various mass equations $M\left(A, T_{z}\right)$ and solutions $M^{*}\left(A, T_{z}\right)$ as functions of $T_{z}$ or $A$. The effects of the different extrapolation is seen more clearly if one of the equations is used as a reference equation and the quantity $M-M_{\text {ref }}$ is plotted.

Figs. 3a-3f show plots of the quantity $M-M_{\text {ref }}$ as a function of $T_{z}$ for an arbitrarily chosen value of $A=100$. The reference equation is MS-C. The star symbols represent the experimental mass values. For clarity the curves for the various equations are subdivided into six groups. Fig. 3a shows six liquid-drop model equations. As expected for few-parameter equations, they agree with the experimental masses only within a few $\mathrm{MeV}$. They diverge from each other particularly on the neutron-rich side and for $T_{z}<0$. Fig. $3 \mathrm{~b}$ shows as an example the comparison between the dropletmodel equation MS and the solutions MS-A, MS-B and MS-C (which is the horizontal line). As expected, MS-A, MS-B and MS-C agree much better with the experimental masses than MS. The extrapolated predictions from MS-A deviate markedly from MS-B and MS-C. The latter solutions, obtained with the constraints $\Delta M_{\text {Coul }} \equiv 0$ or $\Delta M_{\text {sym }} \equiv 0$, respectively, remain quite close to each other one the neutron- and the proton-rich side. This behavior persists for all values of $A$ as can be seen below in fig. $4 \mathrm{~b}$. Fig. $3 \mathrm{c}$ shows the results for GK-T, GK-L, CON, LZ1 and LZ2. While describing the experimental values well, the divergence of the various predictions on the neutron- and proton-rich side is quite significant. The solution CON should not be taken seriously. Clearly, a symmetry energy term of the form $\eta_{1}(N-Z)^{2}$ will grossly underestimate the true symmetry energy for light nuclei and overestimate it for heavy nuclei. Figs. $3 \mathrm{~d}, 3 \mathrm{e}$ and $3 \mathrm{f}$ show the results for the various solutions of the type A, B and C, respectively. Again, the extrapolations diverge considerably, particularly for $B$ and $C$. It is worth noting that the liquid-drop equation $S$ of Seeger ${ }^{12}$ ) leads to the most consistent solutions S-A, S-B and S-C. This result is supported by the fact that $\eta_{1}$ has the smallest value of all solutions $A$ (liquid drop only) which results in practically the same deviation $\sigma_{\mathrm{m}}$ for S-A and S-C (it is also the smallest).

Figs. $4 \mathrm{a}-4 \mathrm{~d}$ show plots of the quantity $M-M_{\text {ref }}$ as a function of $A$ for nuclei 

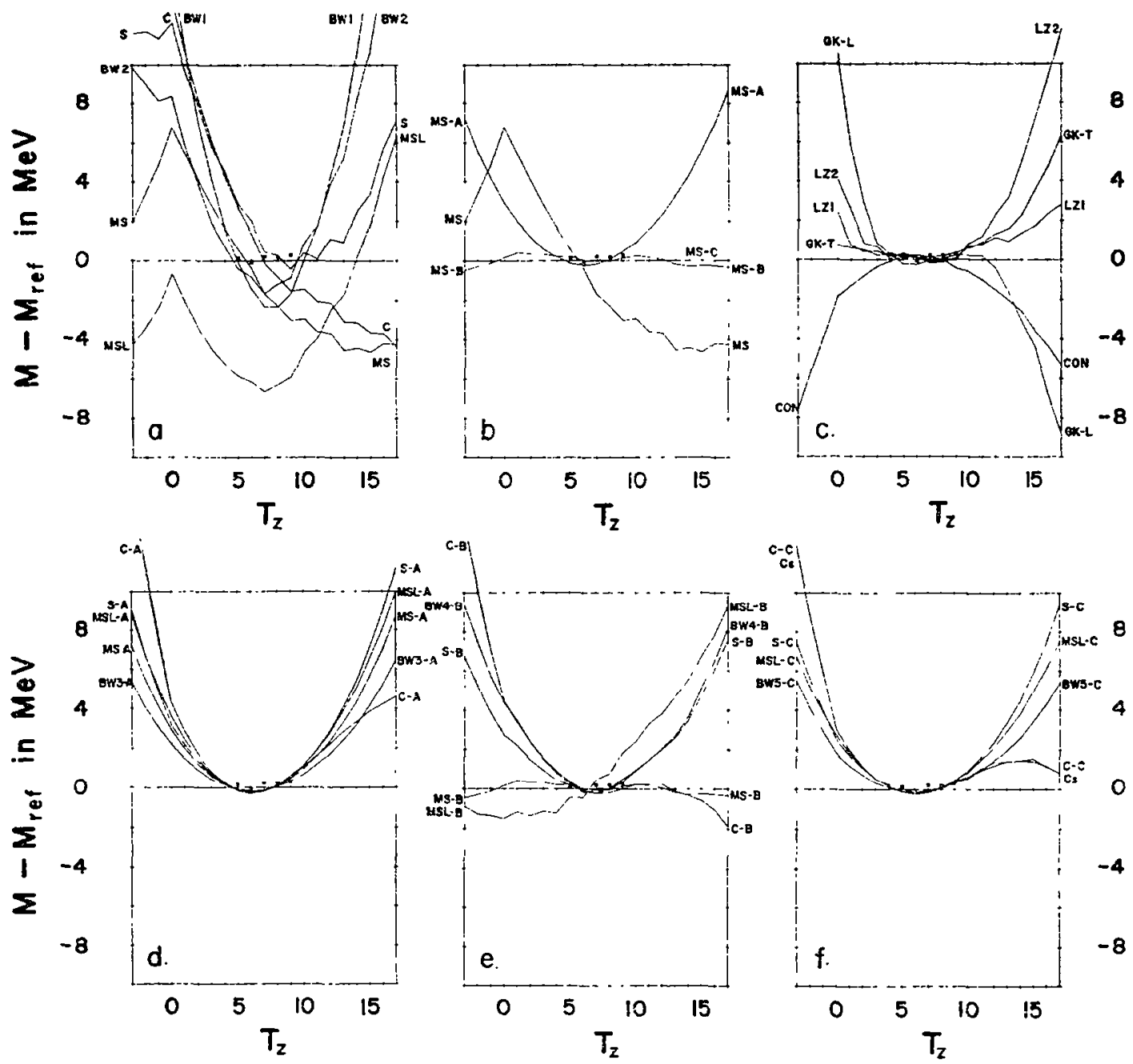

Fig. 3. Plot of the difference $M-M_{\text {ref }}$ as a function of $T_{z}$ for nuclei with $A=100$. The reference equation is MS-C. The star symbols represent the experimental mass values. The lines represent various mass equations and solutions of nuclidic mass relationships. (a) BW1, BW2, MS, MSL, C, S. (b) MS, MS-A, MS-B. (c) GK-T, GK-L, CON, LZ1, LZ2. (d) BW3-A, MS-A, MSL-A, C-A, S-A. (e) BW4-B, MS-B, MSL-B, C-B, S-B. (f) BW5-C, MSL-C, Cs, C-C, S-C.

along the line of $\beta$-stability defined by

$$
T_{z}=\frac{0.2 A^{2}}{200+A}
$$

and for nuclei which are located distances of $\Delta T_{z}= \pm 3$ and $\Delta T_{z}= \pm 6$ away from this line on the proton-rich and the neutron-rich side. The star symbols represent the experimental mass values. The reference equation for fig. $4 \mathrm{a}$ is MS-C, for $4 \mathrm{~b}-4 \mathrm{~d}$ 

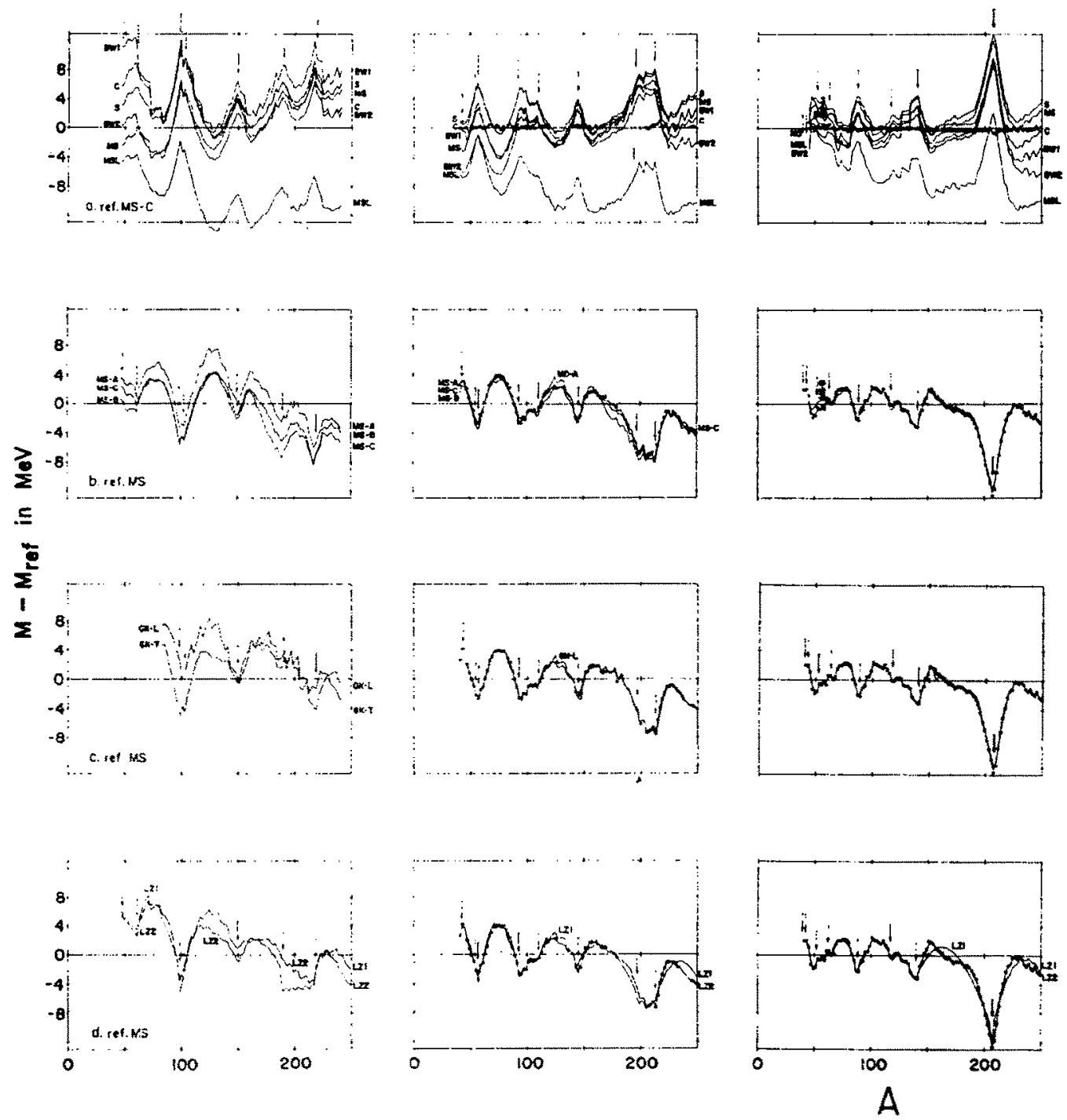

Fig. 4. Plot of the difference $M-M_{\text {ref }}$ as a function of $A$ for nuclei along the line of $\beta$-stability and fig. $4 \mathrm{a}$ is MS-C, for all others it is MS. The star symbols represent the experimental mass values. BW2, MS, MSL, C, S. (b) MS-A, MS-B,

it is MS. Fig. 4a displays all liquid-drop model equations. The positive and negative excursions of several $\mathrm{MeV}$ are due to shell effects. The nucleon numbers $A$ at which shell crossings occur are marked in the figure. Except for MSL, the various predictions are reasonably consistent with each other. The droplet model equation MS was chosen for figs. $4 \mathrm{~b}-4 \mathrm{~d}$ to serve as reference for some of the many-parameter equations. 

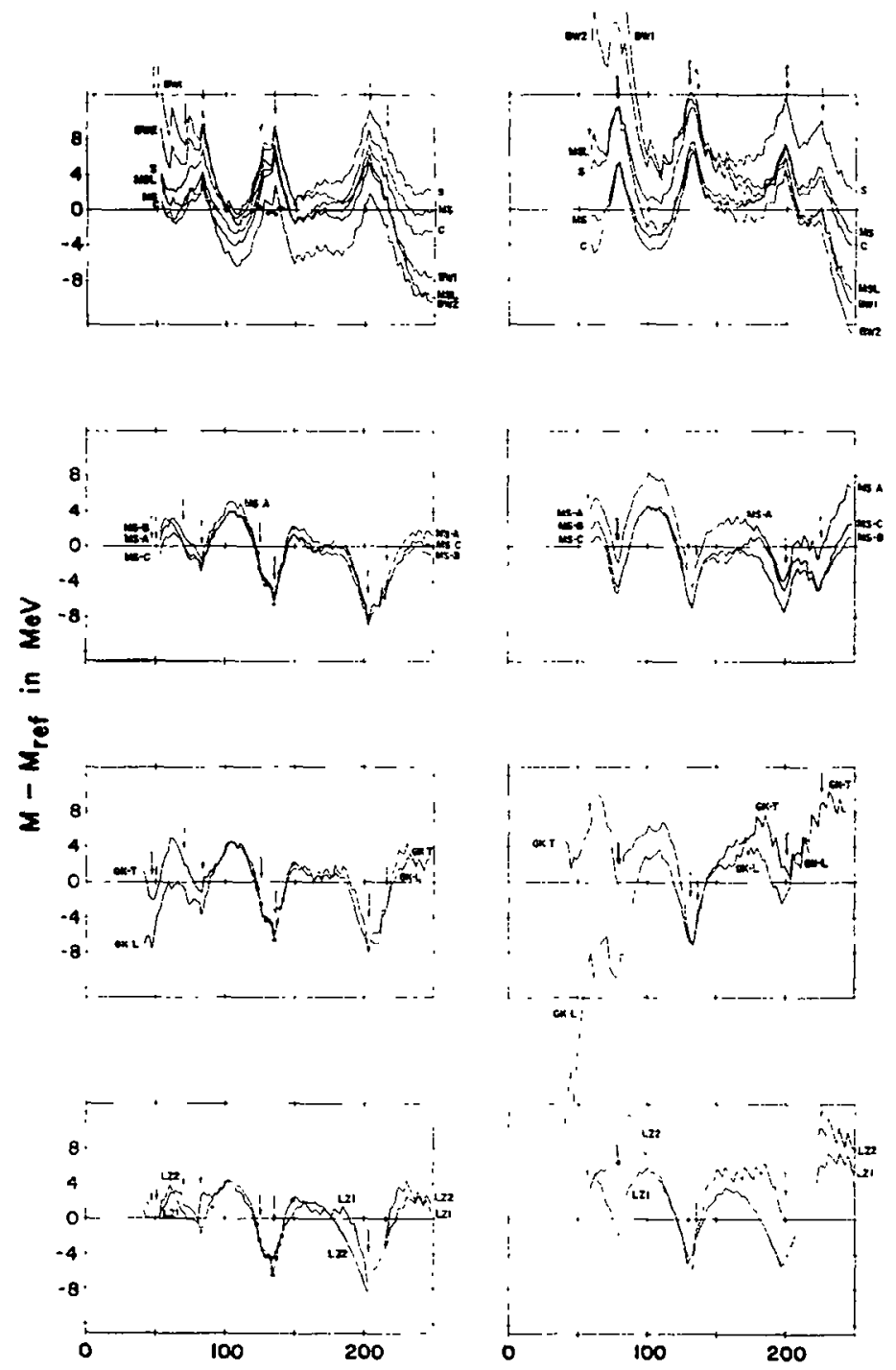

parallel to it, namely $\Delta T_{z}=-6,-3,0,+3,-6$ (from left to right). The reference equation in The lines represent various mass equations and solutions of nuclidic mass relationships. (a) BWI, MS-C. (c) GK-T, GK-L. (d) LZ1, LZ2.

The purpose is to see how the expected shell effects are generated by these equations. Crossings of magic neutron and proton numbers are again indicated. Fig. $4 \mathrm{~b}$ gives the results for MS-A, MS-B and MS-C, fig. $4 c$ for GK-T and GK-L and fig. 4d for LZ1 and LZ2. All equations with the exception of LZ1 in the heavier nuclei reproduce the shell behavior for $\Delta T_{z}=0$ as indicated by the experimental masses 

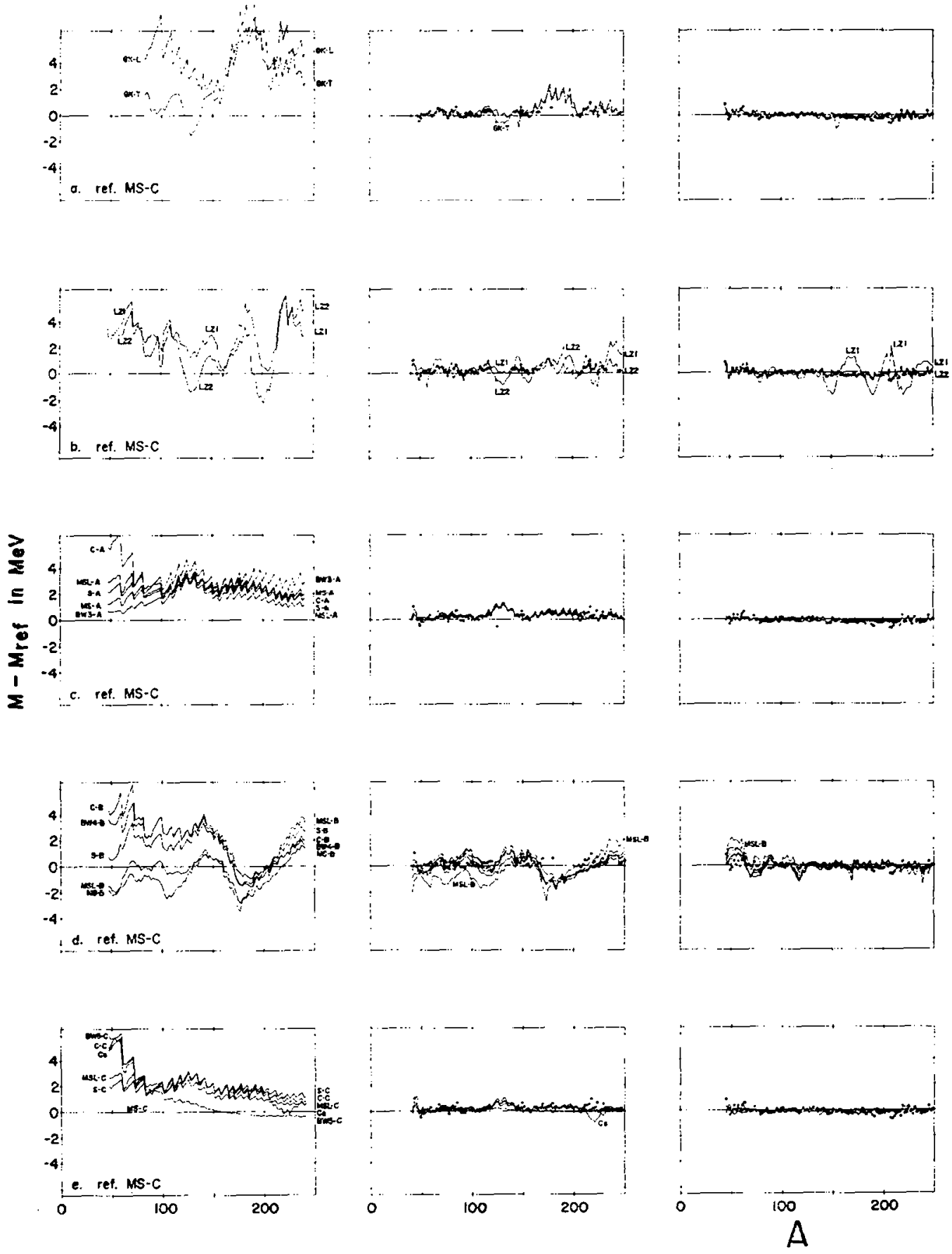

Fig. 5. See text of fig. 4. The reference equation is MS-C. (a) GK-T, GK-L. (b) LZ1, LZ2. (c) BW3-A, 

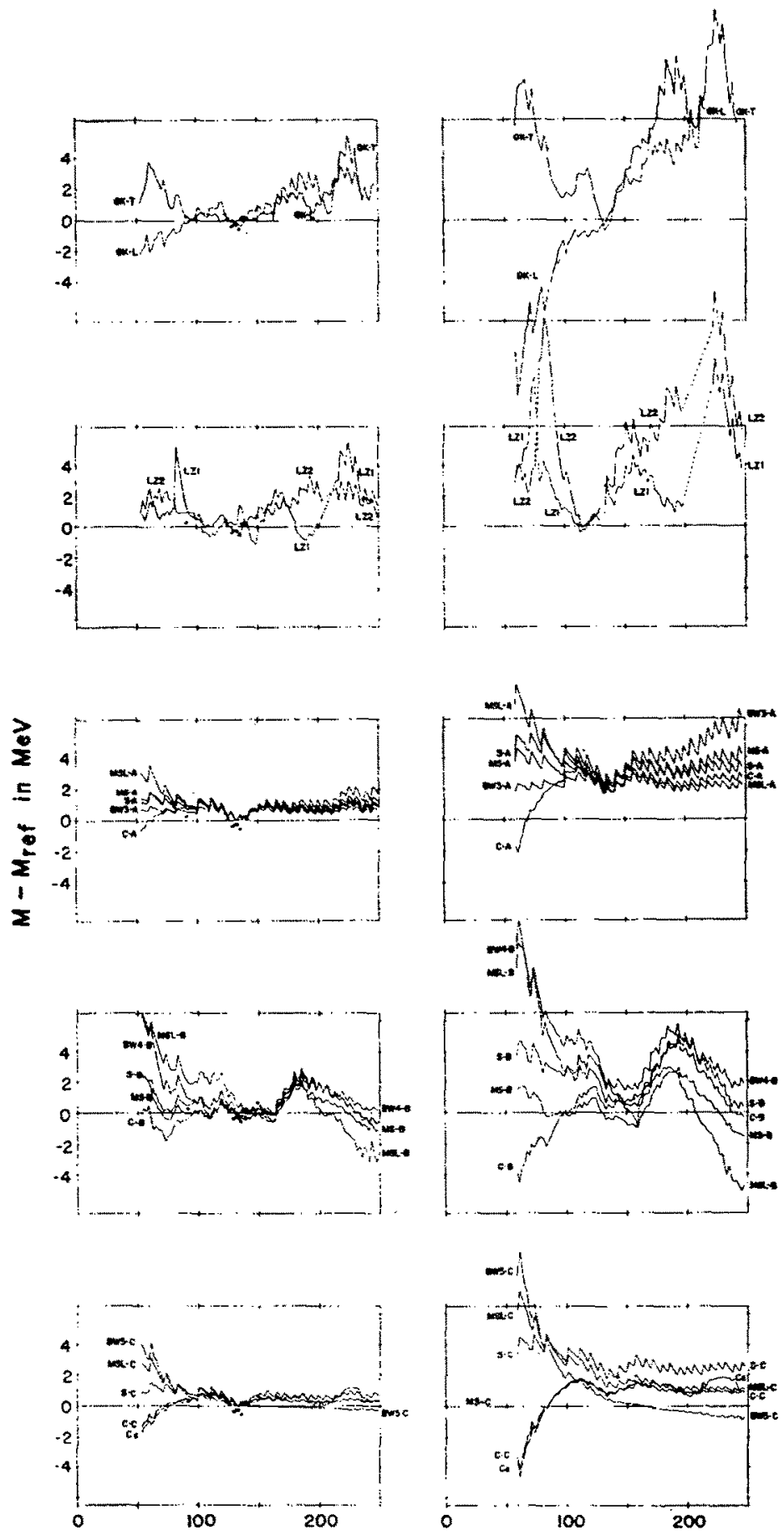

MS-A, MSL-A, C-A, S-A. (d) BW4-B, MS-B, MSL-B, C-B, S-B. (e) BW5-C, MS-C,MSL-C, Cs, C-C,- SC. 
remarkably well. The limited data for $\Delta T_{z}= \pm 3$ are also well reproduced. Clearly the same kind of behavior must persist into the more neutron-rich and proton-rich regions. The sharp minimum near ${ }^{208} \mathrm{~Pb}$, for example, which results from the crossing of the $N=126$ and $Z=82$ lines must separate into two minima (deeper for $N=126$ ) away from the line of $\beta$-stability. Indeed, the predictions MS-A, MS-B and MS-C in fig. $4 \mathrm{~b}$ display the expected behavior quite well. On the other hand minima from the crossing of the $Z=82$ line are not apparent in GK-T, GK-L, LZ2 and to a certain extent LZ1. Similar deficiencies but not quite as pronounced occur at other shell crossings. The predictions GK-L below $A=80$ are much lower than the droplet model predictions MS. It is concluded from fig. 4 that the solutions of generalized nuclidic mass relationships based on an effective neutron-proton interaction $I_{\mathrm{np}}$ derived from liquid-drop models are very adequate in describing the shell behavior near and away from the line of $\beta$-stability.

Figs. 5a-5e show plots of the quantity $M-M_{\text {ref }}$ as a function of $A$ similar to fig. 4 . The scale is expanded by a factor of two. The reference equation for all plots is MS-C. The equations considered are essentially those of figs. $3 c-3$. The oscillatory behavior which is particularly apparent in figs. $5 c-5 e$ is easy to understand. It is the result of small differences in the symmetry energy coefficients which leads to discontinuous steps whenever $T$ changes. The predictions for very neutron-rich and proton-rich nuclei differ considerably. Particularly the differences in the behavior of GK-T, GK-L, LZ1 and LZ2 on the one hand and of the solutions of type A, B and $\mathrm{C}$ on the other are quite striking.

The predictions from MS-C (the line $M-M_{\text {ref }} \equiv 0$ ) are generally lower than those from most other mass equations. The predictions from S-C represent more centered and more balanced estimates. It was pointed out earlier that $\mathrm{S}-\mathrm{C}$ prescnts the most consistent solution of type $\mathrm{C}$ and that it has the smallest standard deviation $\sigma_{\mathrm{M}}$. At present, the solution S-C is therefore considered to yield the most reliable predictions for the masses of unknown neutron-rich and proton-rich nuclei. It is hoped that this statement will be supported by future mass measurements and by other considerations.

It is believed that all mass equations $M\left(A, T_{z}\right)$ and solutions $M^{*}\left(A, T_{z}\right)$ (including $\mathrm{S}-\mathrm{C}$ ) of the generalized nuclidic mass relationships have short-comings. The most important one is probably the fact that the effective neutron-proton interaction $I_{\mathrm{np}}$ underlying the solutions $M^{*}\left(A, T_{z}\right)$ is very simple-minded. This deficiency has to be absorbed by the functions $F_{1}(N)$ and $F_{2}(Z)$ and may be responsible for the slight misrepresentation of the Coulomb energies. Shell-dependent symmetry energy coefficients are definitely required, and their decrease within a given shell with increasing $A$ due to the increase of the matter and potential radii has to be studied carefully. Correlations between neutrons and protons in strongly deformed nuclei will probably also affect $I_{\text {up }}$. The neutron-proton pairing energy appears to depend on isospin $T$ as was observed earlier ${ }^{2}$ ). More attention has to be given to these more refined effects and more realistic expressions will have to be considered in the future, particularly for light nuclei. 


\section{Many-parameter mass equations for $A \geqq 70$}

Numerical values for the coefficients of the many-parameter equations S-C and MS-C will be given below. Both are solutions of generalized nuclidic mass relationships obtained under the constraint $\Delta M_{\text {sym }}=0$ with liquid-drop model representations of the effective neutron-proton interaction $I_{\mathrm{np}}$. The former solution is based on $I_{\mathrm{np}}$ from the equation of Seeger ${ }^{12}$ ). It is believed to result in reliable estimates for masses of unknown nuclei because of the internal consistencies pointed out earlier. The standard deviation of S.C for reproducing the known experimental masses is $\sigma_{\mathrm{m}}=263 \mathrm{keV}$, the standard deviation for reproducing the experimental Coulomb displacement energies is $\sigma_{\mathrm{C}}=420 \mathrm{keV}$. The second equation is based on $I_{\mathrm{np}}$ from the equation of Myers and Swiatecki ${ }^{8,9}$ ). It is believed to slightly underestimate the masses of neutron-rich and proton-rich nuclei. Nevertheless, it is presented here because the droplet model contains the best justified description of macroscopic properties of nuclei. The standard deviation of MS-C for reproducing the known masses is $\sigma_{\mathrm{m}}=320 \mathrm{keV}$, the standard deviation for reproducing the experimental Coulomb displacement energies is $\sigma_{\mathrm{C}}=399 \mathrm{keV}$. Neither equation should be used below $A=70$ because of the diverging predictions (see figs. $5 \mathrm{c}-5 \mathrm{e}$ ) in this region.

The solution S-C can be written as $M^{*}\left(A, T_{z}\right)=M\left(A, T_{z}\right)+\Delta M\left(A, T_{z}\right)$ with a binding energy expression ${ }^{12}$ )

$$
\begin{gathered}
B(N, Z)=\alpha A-\beta \frac{(N-Z)^{2}}{A}-\gamma A^{\frac{3}{3}}+\eta \frac{(N-Z)^{2}}{A^{3}}-864 \frac{Z^{2}}{r_{0} A^{4}}\left\{1-\frac{0.76361}{Z^{3}}-\frac{2.453}{r_{0}^{2} A^{3}}\right\} \\
\pm \delta \frac{1}{\sqrt{3} A}+7000 \exp \left(-6 \frac{(N-Z)}{A}\right)+14.33 \times 10^{-3} Z^{2.39}
\end{gathered}
$$

The coefficients are

$$
\begin{array}{llrl}
\alpha=15971.3 \mathrm{keV}, & \beta=30047 \mathrm{keV}, & \gamma=20806 \mathrm{keV}, \\
\eta=45350 \mathrm{keV}, & \delta=(9600) \mathrm{keV}, & r_{0}=1.16552 \mathrm{fm} .
\end{array}
$$

The term $\Delta M\left(A, T_{z}\right)$ is that of eq. (7) with $\eta_{1}=0 \mathrm{keV}, \eta_{2}=\eta_{3}=-109.8 \mathrm{keV}$, $\eta_{4}=\eta_{5}=0 \mathrm{keV}$ and $F_{1}(N)$ and $F_{2}(Z)$ from table 4 . The coefficient $\delta$ has to be set equal to zero if the term $\Delta M$ is included.

The solution MS-C can be written as $M^{*}\left(A, T_{z}\right)=M\left(A, T_{z}\right)+\Delta M\left(A, T_{z}\right)$ with binding energy expression ${ }^{8,9}$ )

$$
\begin{aligned}
-B(N, Z) & =\left[-a_{1}+J \delta^{2}-\frac{1}{2} K \bar{\varepsilon}^{2}+\frac{1}{2} M \delta^{4}\right] A+\left[a_{2}+\frac{9}{4}\left(J^{2} / Q\right) \delta^{2}\right] A^{\frac{3}{3}}+a_{3} A^{\frac{3}{3}} \\
& +c_{1} Z^{2} A^{-\frac{1}{3}}-c_{2} Z^{2} A^{\frac{1}{3}}-c_{5} Z^{2}-c_{3} Z^{2} A^{-1}-\left(c_{4} / 2^{3}\right) Z \pm a_{\mathrm{p}} A^{-\frac{1}{2}}+a_{\mathrm{w}}|I|,
\end{aligned}
$$

with

$$
\begin{gathered}
\tilde{\delta}=\left[I+\frac{3}{16}\left(c_{1} / Q\right) Z A^{-\frac{3}{3}}\right] /\left[1+\frac{9}{4}(J / Q) A^{-\dagger}\right], \\
\bar{\varepsilon}=\left[-2 a_{2} A^{-\frac{3}{3}}+L \bar{\delta}^{2}+c_{1} Z^{2} A^{-5}\right] / K,
\end{gathered}
$$

$I=(N-Z) /(N+Z)$. 


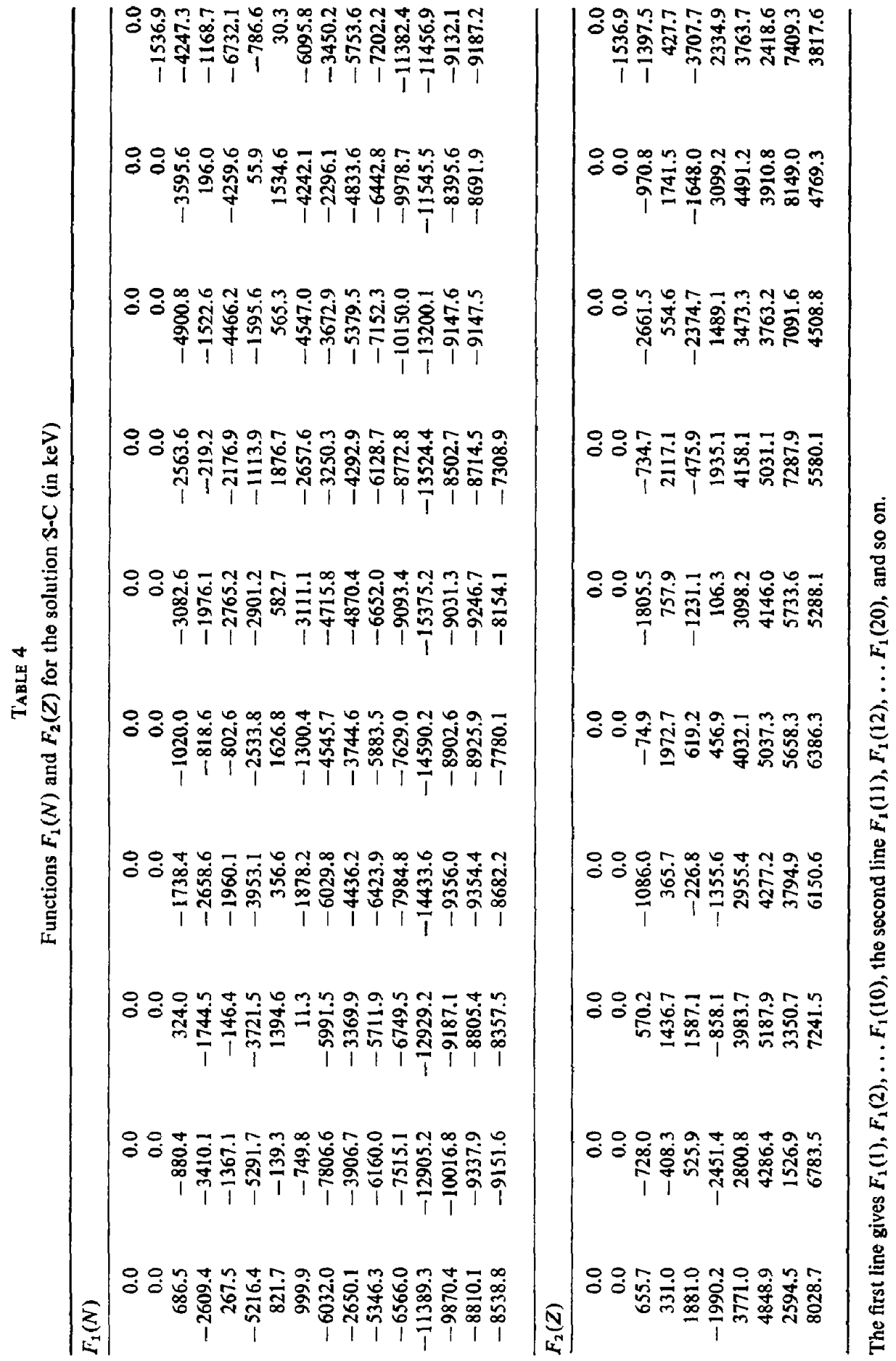




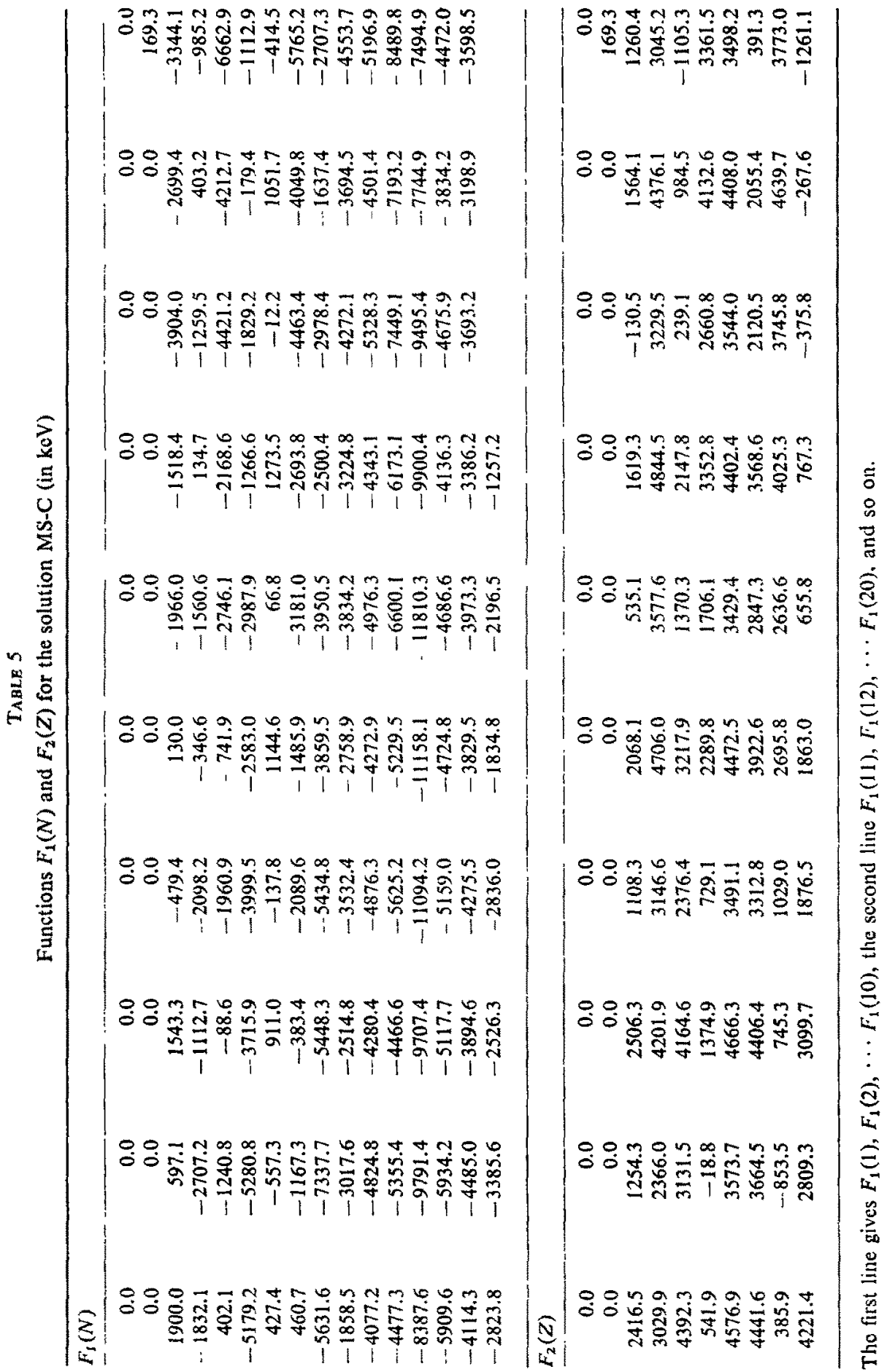


The coefficients are (in $\mathrm{keV}$ )

$$
\begin{array}{rlrl}
a_{1}=15986, & c_{1}= & 735.31, \\
a_{2}=20760, & c_{2}= & 0.16477, \\
a_{3}=00, & c_{3}= & 1305.01, \\
J & =36500, & & c_{4}=561.49, \\
Q=17000, & c_{5}= & 0.49695, \\
K & =240000, & a_{\mathrm{p}}=(11000), \\
L=100000, & a_{\mathrm{w}}=30000 . \\
M=0, &
\end{array}
$$

The term $\Delta M\left(A, T_{z}\right)$ is that of eq. (7) with $\eta_{1}=0 \mathrm{keV}, \eta_{2}=\eta_{3}=-120.1 \mathrm{keV}$, $\eta_{4}=\eta_{5}=0 \mathrm{keV}$ and $F_{1}(N)$ and $F_{2}(Z)$ from table 5 . The coefficient $a_{p}$ has to be set equal to zero if the term $\Delta M$ is included.

Thanks are due to F. L. Milder and R. R. Howell for considerable help with computer programming. A research grant from the Michigan Memorial Phoenix Project, The University of Michigan, is highly appreciated.

\section{References}

1) J. Jänecke and H. Behrens, Z. Phys. 256 (1972) 236

2) J. Jânecke and H. Behrens, Phys. Rev. C9 (1974) 1276; Proc. Int. Conf. on nuclear physics, Munich, 1973, vol. 2, p. 321

3) G. T. Garvey, W. J. Gerace, R. L. Jaffe, I. Talmi and I. Kelson, Rev. Mod. Phys. 41 (1969) SI

4) S. Liran and N. Zeldes, Proc. Int. Conf. on nuclear physics, Munich, 1973, p. 322;

S. Liran, Ph.D. thesis, 1973, Hebrew University of Jerusalem; and private communication

5) N. Zeldes, A. Grill and A. Simievic, Mat. Fys. Skr. Dan. Vid. Selsk. 3 (1967) no. 5

6) J. Jänecke, Phys. Rev. C6 (1972) 467

7) J. Jänecke, in Isospin in nuclear physics, ed. D. H. Wilkinson (North-Holland, Amsterdam, 1969) p. 298

8) W. D. Myers and W. J. Swiatecki, Ann. of Phys. 55 (1969) 395

9) W. D. Myers and W. J. Swiatecki, Ann. of Phys., to be published; report LBL-1957; and private communication

10) S. Ludwig, H. von Groote, E. Hil, A. G. W. Cameron and J. Truran, Nucl. Phys. A203 (1973) 627

11) J. W. Truran, A. G. W. Cameron and E. Hilf, Proc. Int. Conf, on propertics of nuclei far from the region of beta-stability, Leysin, 1970, vol. 1, p. 275

12) P. A. Seeger, ibid., p. 217

13) A. H. Wapstra and N. B. Gove, Nucl. Data A9 (1971) 265; August 1972 version obtained from Oak Ridge National Laboratory

14) R. A. Sorensen, Phys. Lett. 34B (1971) 21

15) J. Jänecke, Phys. Rev. C11 (1975) 625 